\title{
MODELOS NÃO LINEARES NA ANÁLISE DE CURVAS DE PERCENTUAIS GERMINATIVOS DE SEMENTES DE MILHO
}

\section{SEBASTIÃO GAZOLA ${ }^{1}$, CARLOS ALBERTO SCAPIM ${ }^{1}$, ALESSANDRO LUCCA BRACCINI ${ }^{1}$, ANTONIO TEIXEIRA DO AMARAL JÚNIOR ${ }^{2}$ e MARCELO VIVAS ${ }^{2}$}

'Universidade Estadualde Maringá,sgazola@uem.br; cascapim@uem.br; albraccini@uem.br;

${ }^{2}$ Universidade Estadual do Norte Fluminense Darcy Ribeiro, amaraljr@pq.cnpq.br; mrclvivas@hotmail.com

Revista Brasileira de Milho e Sorgo, v.16, n.1, p. 142-152, 2017

RESUMO - O objetivo do presente trabalho foi investigar a resposta de germinação de sementes, em função do número de dias após o florescimento feminino, para os híbridos de milho OC 705 e CD 5501, em duas épocas de semeadura. Ajustou-se o modelo de regressão não linear $\mathrm{y}(\mathrm{t})=\mathrm{A}-\mathrm{B}$.exp $(-\mathrm{C} . \mathrm{t})$ aos conjuntos de percentuais germinativos, combinando híbridos e épocas. Para avaliar a curva do percentual de germinação das sementes dos híbridos nas diferentes épocas, aplicou-se o teste de hipóteses para testar a identidade de equações de regressão não lineares utilizando-se as estatísticas $\chi^{2}$ e F. As medidas de curvatura e viés de Box indicaram que o modelo se ajustou adequadamente aos dados. Pelos testes de comparação, apenas a primeira época de semeadura apresentou o percentual de germinação das sementes dos dois híbridos descritos por uma única equação. Verificou-se que a primeira época foi a mais adequada para a semeadura dos dois híbridos, devendo a colheita ser realizada 57 dias após o florescimento feminino para obter o percentual máximo de $96,1 \%$ de germinação das sementes.

Palavras-chave: Zea mays L., regressão não linear, ajuste de percentuais germinativos, comparação de equações.

\section{DESCRIBING THE PERCENTAGE OF GERMINATION OF MAIZE SEEDS UNDER MATURATION FITTING NONLINEAR PARAMETERS}

\begin{abstract}
The objective of this experiment was to describe the seed germination of the maize hybrids OC 705 and CD 5501 during the maturation period, and using parameters of nonlinear models. These genotypes were sowed in two dates in the growing season. We tested the identity of the models by the $\chi^{2}$ and $\mathrm{F}$ tests. We tested the goodness of fit by the IN and PE effect curvatures, and the bias of Box. The identity of the models indicated that only the first sowing time had the responses from both maize hybrids described by the parameters of the nonlinear model $\mathrm{y}\left(\mathrm{t}_{\mathrm{i}}\right)=\mathrm{A}-\mathrm{B}_{*} \exp \left(-\mathrm{C}_{*} \mathrm{t}_{\mathrm{i}}\right)$. The first sowing date had the best seed germination responses. Fifty-seven days after the anthesis is the most suitable maturation period for seed harvesting because the estimate of the maximum percentage of seed germination was $96.1 \%$.
\end{abstract}

Keywords: Zea mays L., nonlinear regression, adjust of percentile germinate, model identity. 
Em estudo da viabilidade de sementes, os dados nem sempre podem ser representados por um modelo linear, a exemplo de um modelo polinomial de grau k. Quando se estuda um fenômeno ao longo do tempo, observa-se um crescimento mais acentuado no início e que vai se estabilizando no final. Um modelo de regressão linear não tem o poder de captar esse comportamento, sendo, então, mais conveniente buscar uma representação desses tipos de dados por funções não lineares. Segundo Bates e Watts (1988), em algumas situações existe mais do que uma função que pode ser usada e, nesse caso, muitas vezes tem-se interesse em encontrar simplesmente um modelo que se ajuste adequadamente aos dados.

Regazzi (2003) apresentou um método para testar a identidade de modelos não lineares. Ele foi baseado no teste da razão de verossimilhança, que para grandes amostras tem estatística qui-quadrado $\left(\chi^{2}\right)$. O autor ilustrou o método com dados de matéria seca total na cultura do milho. Ainda utilizando a mesma metodologia, Regazzi e Silva (2004) a aplicaram em dados de velocidade de reação versus concentração de substrato de um experimento com Puromycin. Guimarães et al. (2006) ajustaram vários modelos não lineares a dados de produção de leite ao longo do tempo de lactação (em semanas). Os autores aplicaram o teste de igualdade de modelos para a curva de lactação de diferentes raças, utilizando a estatística $\chi^{2}$.

Carvalho e Christoffoleti (2007) ajustaram o modelo de regressão não linear do tipo Logístico, proposto por Streibig em 1988, para avaliar a influência da luz (fotoperíodo ou escuro) e da temperatura (alternância ou constância) na germinação das sementes de cinco espécies de plantas daninhas do gênero Amaranthus. Sánchez-Coronado et al. (2007) ajustaram a curva exponencial sigmoide $\mathrm{y}=\mathrm{a} /(1+\mathrm{b}(-\mathrm{ct}))$ para estudar o percentual de germinação, ao longo do tempo (dias), de sementes de Omphalea oleifera (Euphorbiaceae). Nogueira et al. (2008) estudaram o efeito do afastamento dos fustes de árvores de Pinus taeda, ajustando modelos não lineares e aplicando o teste de identidade de modelos. Siqueira et al. (2009) ajustaram os modelos não lineares Brody, Gompertz, Logístico, von Bertalanffy e concluíram que o modelo Logístico apresentou melhor qualidade de ajuste e pode ser utilizado para descrever curvas de crescimento de bananeiras. Rizzardi et al. (2009) estudaram os processos de germinação das sementes e elongação das plântulas de corda-de-viola, em termos do tempo hidrotérmico e temperatura, respectivamente, utilizando o modelo não linear de Weibull.

Por sua vez, na germinação de sementes, Cardoso e Pereira (2008) ajustaram o modelo de Weibull, $\Psi=\mathrm{A}\{1-\exp [-\mathrm{k}(\mathrm{x}-\mathrm{z}) \mathrm{d}]\}$ para descrever a distribuição das porcentagens acumuladas de germinação de sementes de Drymaria cordata ao longo do tempo de semeadura em dias. Cardoso e Pereira (2008) elaboraram, para diversas temperaturas, curvas de distribuição das porcentagens médias de germinação acumulada ao longo do tempo (dias), sendo os pontos ajustados de acordo com a função de Weibull, obedecendo à equação $\mathrm{g}=\mathrm{A}[1-\exp (-\{\mathrm{k}[\mathrm{t}-\mathrm{z}]\})]$.

Nos experimentos de germinação de sementes ao longo do tempo, é de interesse do pesquisador identificar o potencial de germinação de diferentes lotes de sementes, híbridos e/ou épocas e investigar se a combinação de híbridos e épocas pode ser representada por uma única equação. Assim, os objetivos deste trabalho foram: (i) verificar o percentual de germinação de sementes de milho, em função dos dias após o florescimento feminino, para a combinação de dois híbridos e duas épocas de semeadura; (ii) verificar, por meio da teoria de identidade de modelos 
não lineares, se diferentes híbridos de milho combinados com diferentes épocas de semeadura podem ser descritos por uma única equação; e (iii) determinar o período (dias) mais favorável de colheita dos híbridos para cada época de semeadura.

\section{Material e Métodos}

O trabalho foi conduzido na Universidade Estadual de Maringá (UEM), em Maringá-PR, em conjunto com a Cooperativa Central de Pesquisa Agrícola (Coodetec) e conduzido na Fazenda Experimental da Coodetec, em Cascavel, Estado do Paraná, situado à latitude $24^{\circ} 56^{\prime} \mathrm{S}$, longitude $53^{\circ} 26^{\prime} \mathrm{W}$ e altitude média de 760 m, em Latossolo Roxo distrófico. O experimento consistiu na semeadura dos híbridos de milho OC 705 (duplo) e CD 5501 (simples), em duas épocas de semeadura. As épocas foram as seguintes:

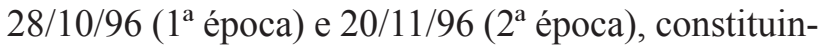
do-se um ensaio em cada uma.

Empregou-se, em cada época de semeadura, o delineamento inteiramente casualizado, com os tratamentos dispostos em parcelas subdivididas no tempo. Nas parcelas foram casualizados os dois híbridos de milho (OC 705 e CD 5501) e nas subparcelas foram tomadas dez avaliações consecutivas (períodos de amostragem), correspondendo a intervalos de quatro dias, com quatro repetições. Essas amostragens foram iniciadas aos 23 dias após o florescimento feminino, sendo a última amostragem coletada aos 59 dias após esse período.

Cada parcela foi constituída por quatro linhas de doze metros de comprimento e espaçamento de $0,9 \mathrm{~m}$ entre linhas, perfazendo um total de $43,2 \mathrm{~m}^{2}$ de área. Foi descartado um metro de cada extremidade das fileiras, além da primeira e da quarta linha lateral das parcelas como bordaduras, perfazendo um total de $18 \mathrm{~m}^{2}$ de área útil. Neste trabalho, abandonou-se a estrutura de delineamento e fez-se a análise de identidade de equações.

Quando da ocorrência dos primórdios das espigas, plantas com aparência uniforme foram selecionadas e marcadas com etiquetas presas ao colmo para serem posteriormente amostradas. Quando 75\% das plantas a serem amostradas na parcela apresentavam o estigma com aproximadamente 2,5 centímetros de comprimento, foi considerado como início da floração.

As amostras em 34 espigas por parcela consistiram de 20 a 25 sementes retiradas de cada espiga na sua porção mediana e de uma mesma fileira de sementes da espiga. Para a retirada da amostra de cada espiga foi necessária a utilização de uma lâmina apropriada para cortar a palha e uma faca devidamente preparada para auxiliar na retirada das sementes das espigas. Após a retirada da amostra de uma espiga, esta era coberta por um saco de papel, que era preso por um elástico. As sementes foram selecionadas e encaminhadas ao laboratório para a análise do teste de germinação. Esse teste foi conduzido utilizando duas repetições analíticas de cinquenta sementes, para cada repetição de campo. As amostras foram colocadas entre três folhas de papel toalha (germitest), previamente umedecidas com água desmineralizada, na proporção de 2,5 vezes o peso do substrato seco, sendo confeccionados rolos. Esses foram, então, colocados na posição vertical em germinador do tipo Mangelsdorf regulado para manter a temperatura constante de $25 \pm 1{ }^{\circ} \mathrm{C}$, por um período de sete dias. Ao final desse período, foi realizada a avaliação levando-se em conta os critérios estabelecidos nas Regras para Análises de Sementes (Brasil, 2009), determinando-se a percentagem de plântulas normais e anormais, bem como de sementes mortas. Desta 
forma, combinando-se os híbridos OC 705 (duplo) e CD 5501 (simples) e as épocas de semeadura (época 1 e época 2), obtiveram-se quatro conjuntos de dados: OC705E1, OC705E2, CD5501E1 e CD5501E2.

Em experimentos estudados ao longo do tempo, observa-se uma curva assintótica; assim, é mais conveniente buscar o ajuste desse tipo de dados por meio de funções não lineares. Os conjuntos de dados apresentados anteriormente foram usados para ajuste do modelo não linear de crescimento exponencial, cuja função é dada por: $\mathrm{y}(\mathrm{t})=\mathrm{A}-\mathrm{B} \exp (-\mathrm{Ct})$ ) (Equação 1). O parâmetro A representa a germinação assintótica. $\mathrm{O}$ parâmetro $\mathrm{B}$ está relacionado com o intercepto, e o parâmetro $\mathrm{C}$ representa a taxa média do aumento do percentual de germinação ao longo do tempo.

Para avaliar a qualidade dos resultados dos ajustes do modelo não linear de crescimento exponencial, foram usadas as medidas de curvatura (Bates \& Watts, 1988) e o viés de Box (Ratkowsky, 1983). Para realizar o teste de hipótese de igualdade de equações, foram verificadas as suposições de normalidade de Shapiro Wilk e homocedasticidade de Bartlett's.

Bates e Watts (1988) descreveram formalmente as expressões para o cálculo das medidas de curvatura paramétrica e intrínseca. A curvatura paramétrica mede a não linearidade, em razão do efeito dos parâmetros, e a curvatura intrínseca mede a não linearidade referente ao espaço de estimação.

O valor limite de curvatura sugerido (Bates \& Watts, 1988) é dado por: $\frac{1}{2 \sqrt{F_{1-\alpha,(p, n-p)}}}$ (Equação 2), em que $\mathrm{F}$ é o valor crítico obtido a partir de uma distribuição, $\mathrm{p}$ é o número de parâmetros do modelo e $\mathrm{n}$ é o total de observações. O viés de Box, calculado para cada parâmetro, permite a identificação dos parâmetros que individualmente são responsáveis pelo excesso de curvatura (Souza, 1998). O valor sugerido
(Ratkowsky, 1983) é de 1\%, obtido pela razão entre o valor absoluto do quociente do viés e o valor do parâmetro. O viés permite a identificação dos parâmetros que individualmente são responsáveis pelo excesso de curvatura (Souza, 1998).

Para investigar se a combinação de híbridos e épocas pode ser representada por uma única equação foi necessário aplicar a metodologia de identidade de modelos e, com isso, analisar o efeito dos híbridos e das diferentes épocas de semeadura sobre o percentual de germinação das sementes de milho. Considerando a forma algébrica geral do modelo da função não linear de crescimento exponencial dada na equação (1): $\mathrm{y}_{\mathrm{ij}}(\mathrm{t})=\mathrm{A}_{\mathrm{i}}-\mathrm{B}_{\mathrm{i}} \exp \left(-\mathrm{C}_{\mathrm{i}} \mathrm{t}_{\mathrm{ij}}\right)+\varepsilon_{\mathrm{ij}}$ (equação 3), em que $y_{i j}$ são os valores observados do i-ésimo híbrido e j-ésima observação ( $i=1, \ldots, 2$ e j=1,.., 10), $t_{i j}$ é o tempo em dias, $\mathrm{A}_{\mathrm{i}}$, $\mathrm{B}_{\mathrm{i}}$ e $\mathrm{C}_{\mathrm{i}}$ são os parâmetros desconhecidos e $\varepsilon_{\mathrm{ij}}$ é o erro, sendo suposto independente e normalmente distribuído com média zero e variância constante $\sigma^{2}$.

As hipóteses de interesse para testar a identidade de modelos não lineares, no estudo do potencial de germinação, são descritas por: $\mathrm{H}_{0}: \mathrm{A}_{1}=\ldots=\mathrm{A}_{\mathrm{H}}=\mathrm{A}, \mathrm{B}_{1}=$ $\ldots=\mathrm{B}_{\mathrm{H}}=\mathrm{BeC}_{1}=\ldots=\mathrm{C}_{\mathrm{H}}=\mathrm{C}$, istoé, as Hequações são idênticas. $\mathrm{E}, \mathrm{H}_{\mathrm{a}}$ : pelo menos dois parâmetros diferem entre si, isto é, as $H$ equações não são idênticas. $O$ número total de observações é: $N=\sum_{h=1}^{H} n_{h}$, em que $n_{h}$ é o número de observações de cada conjunto de dados ajustado.

Sob a hipótese de nulidade, ou seja, as equações ajustadas para vários grupos são idênticas, Regazzi (2003) baseia-se no teste da razão de verossimilhança que tem como estatística do teste: $\mathrm{L}=\left(\frac{\hat{\sigma}_{\mathrm{I}}^{2}}{\hat{\sigma}_{\mathrm{U}}^{2}}\right)^{\frac{\mathrm{N}}{2}}$, em que $\sigma_{U}^{2}$ é a estimativa de máxima verossimilhança de $\sigma^{2}$ do modelo ajustado a cada conjunto de dados (modelo individual) e $\sigma_{U}^{2}$ é a estimativa de máxima verossimilhança de $\sigma^{2}$ do modelo ajustado a todos os dados (modelo Geral). 
O logaritmo da equação dada anteriormente fornece: $2 \ln (\mathrm{L})=\operatorname{Nln}\left(\frac{\hat{\sigma}_{\mathrm{I}}^{2}}{\hat{\sigma}_{\mathrm{U}}^{2}}\right)$.

Conforme Rao (1973), para um N suficientemente grande, a distribuição de $-2 \ln (\mathrm{L})$ é, aproximadamente, Qui-quadrado $\left(\chi^{2}\right)$ com v graus de liberdade.

Como $\sigma_{\mathrm{I}}^{2}$ é a soma de quadrado do resíduo da regressão, referente aos modelos individuais, e $\sigma_{\mathrm{U}}^{2}$ é a soma de quadrado do resíduo da regressão, referente ao modelo geral, logo, tem-se: $-2 \ln (\mathrm{L})=-\mathrm{N} \ln \left(\sum_{i=1}^{\mathrm{H}} \mathrm{SQE}_{\mathrm{t}_{i}} / \mathrm{SQE}_{\mathrm{U}}\right)$, em que $\mathrm{SQE}_{\mathrm{I}}$ é a soma de quadrado dos erros do modelo individual e $\mathrm{SQE}_{\mathrm{U}}$ é a soma de quadrado dos erros do modelo geral, que segue, aproximadamente, a distribuição $\chi^{2}$ com $\mathrm{v}$ graus de liberdade, em que $\mathrm{v}$ é a soma do número de parâmetros dos modelos individuais menos o número de parâmetros do modelo geral.

A hipótese $\mathrm{H}_{0}$ é rejeitada se, e somente, se $\chi_{\mathrm{c}}^{2} \geq \chi_{a: v}^{2}$.

Ainda sob a hipótese de nulidade, com base no teste da razão de verossimilhança e usando as estimativas de máxima verossimilhança dos parâmetros, pode-se usar a estatística F com $(\mathrm{H}-1)$ p e $(\mathrm{N}-\mathrm{Hp})$ graus de liberdade, em que H é o número de equações e p é o número de parâmetros do modelo (Bates \& Watts, 1988).

O cálculo do valor de $\mathrm{F}$ é obtido pela expressão:

$$
F=\frac{\left(\mathrm{SQE}_{\mathrm{U}}-\sum_{\mathrm{i}=1}^{\mathrm{H}} \mathrm{SQE}_{\mathrm{i}_{\mathrm{i}}}\right) /(\mathrm{H}-1) \mathrm{p}}{\left.\sum_{\mathrm{i}=1}^{H} \mathrm{SQE}_{\mathrm{i}_{1}} / \mathrm{N}-\mathrm{Np}\right)} \text {. }
$$

A hipótese $\mathrm{H}_{0}$ é rejeitada se, e somente se, $\mathrm{F}_{\mathrm{C}} \geq \mathrm{F}_{\alpha:(\mathrm{H}-1) \mathrm{p} ;(\mathrm{N}-\mathrm{Hp})}$

\section{Resultados e Discussão}

Os conjuntos de valores de porcentagem de germinação dos híbridos OC705E1 (OC 705 - duplo, na época 1), CD5501E1 (CD 5501 - simples, na época 1), CD5501E2 (CD 5501 - simples, na época 2) e OC705E2 (OC 705 - duplo, na época 2) foram ajustados pelo modelo de crescimento exponencial, conforme equação (1). Ajustaram-se os dois híbridos, fixando-se as épocas, para avaliação de cada época (Figura 1). Ajustou-se, também, a equação (1), para os conjuntos de dados geral da época um (OC705E1UCD5501E1) e geral da época dois (OC705E2UCD5501E2). Embora outros trabalhos já tenham sido conduzidos visando avaliar o vigor de sementes de milho (Gazola et al., 2015, 2016), este é inédito na interpretação do parâmetro $\mathrm{C}$ da equação de regressão do modelo não linear proposto.

Nas duas épocas de semeadura (Tabela 1), as medidas de curvatura intrínseca indicaram forte presença de linearidade (Bates \& Watts, 1988) nas duas equações. Apenas as medidas de curvatura paramétrica foram significativas. O viés de Box indicou que o parâmetro responsável pelo afastamento da linearidade é apenas o parâmetro B (Ratkowsky, 1983). O pvalor do teste de Shapiro Wilk indicou a não violação da suposição de normalidade para todas as equações ( $p>0,05)$ e o teste de Bartlett's para as equações completas mostrou a não violação da suposição de homocedasticidade ( $\mathrm{p}>0,05)$.

O modelo exponencial (equação 1) ajustou-se adequadamente a todos os conjuntos de dados das combinações dos dois híbridos e das duas épocas e, também, para os conjuntos gerais dos dois híbridos de milho. Verificou-se que, para os dois híbridos combinados com as duas épocas de semeadura, o valor da assíntota, parâmetro estimado A, superestimou o percentual máximo de germinação das sementes, variando de 0,97 a 1,0. A taxa média de crescimento, parâmetro $\mathrm{C}$, estimado para todos os híbridos e épocas, variou de 0,07 para o CD5501E2 a 0,11 para o OC705E1. Pela Figura 1 é possível observar que, na época 2, os dois híbridos apresentam curvas esti- 

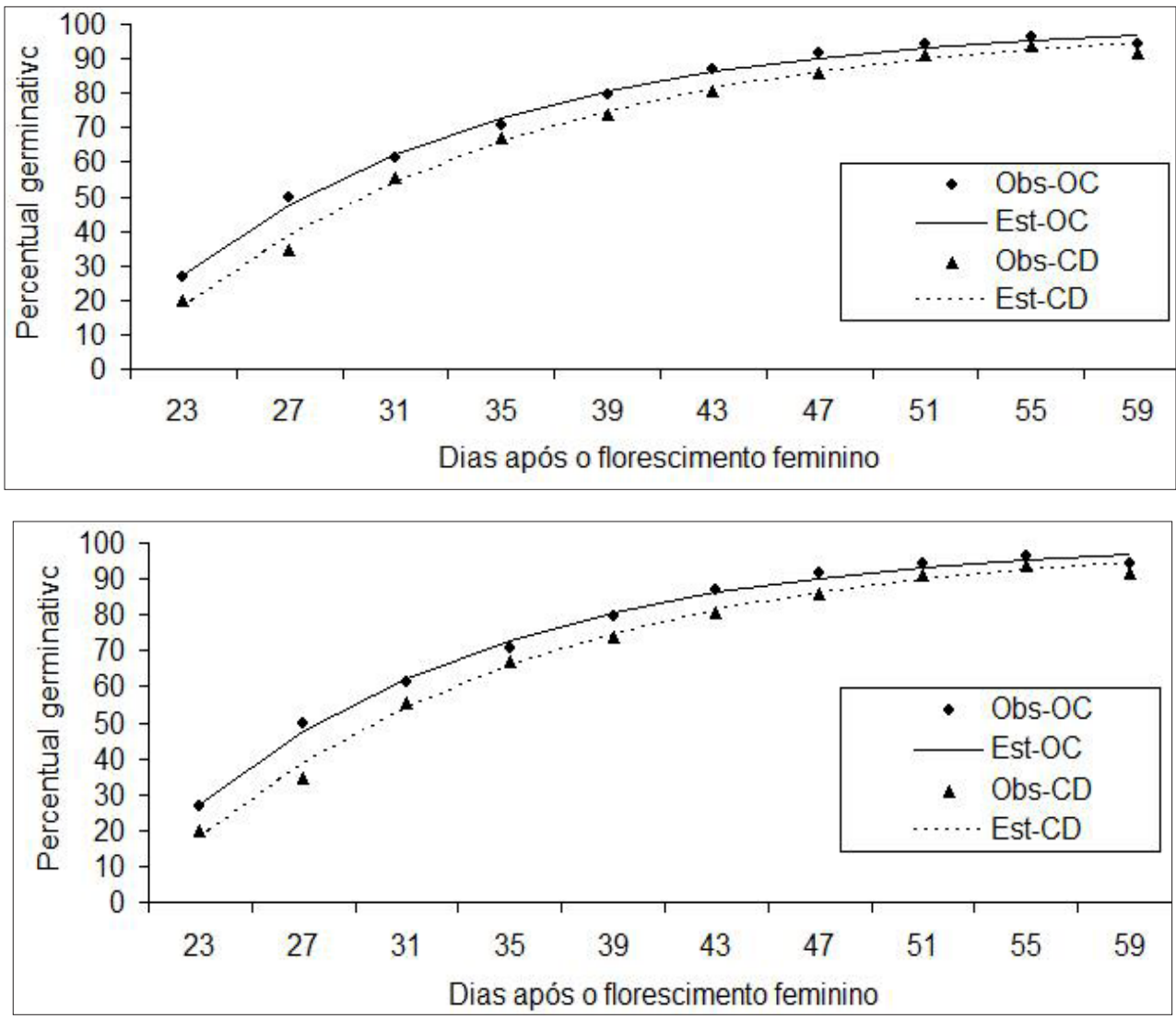

Figura 1. Percentagem de plântulas normais obtidas no teste de germinação das sementes de dois híbridos, em função da época de amostragem, na primeira época de semeadura (E1) e na segunda época de semeadura (E2).

madas com forma menos acentuado que na época 1 ; por isso, a taxa de crescimento médio nessa época foi menor.

Quanto ao parâmetro estimado B, cuja interpretação é apenas matemática, não tendo significado agronômico, os resultados indicaram variação entre os valores estimados, de 4 a 11, isto é por causa da variação existente dos percentuais iniciais, em relação a cada conjunto de dados, pois esse parâmetro está relacionado com o intercepto. Pode-se observar que as curvas referentes aos dois híbridos na época 1 são próximas, enquanto que, para a época 2 , existe um afastamento entre elas (Figura 1).
Foram aplicadas as estatísticas F e Qui-quadrado para verificar se existe igualdade das equações, fixando a época, ou seja, se a equação completa única pode representar o percentual germinativo das sementes dos dois híbridos, ou se realmente deve-se trabalhar com duas equações separadas e tirar conclusões agronômicas para cada híbrido. O teste não rejeitou a hipótese de igualdade para a primeira época de semeadura (E1). Assim, os percentuais germinativos das sementes referentes aos híbridos OC 705 e CD 5501 podem ser representados (ajustados) por uma única equação (Tabela 2). Para a segunda época de semeadura (E2), as duas estatísticas foram significativas, 
ou seja, os híbridos não podem ser representados por uma única equação, devendo, portanto, trabalhar com as equações ajustadas para cada híbrido (Tabela 2).

Além disso, houve interesse de verificar se existe igualdade das equações, fixando o híbrido, ou seja, se uma equação completa única pode representar o percentual germinativo das sementes do híbrido em duas épocas, ou se deve trabalhar com duas equações separadas e tirar conclusões agronômicas para cada época. Para tal, ajustou-se a função do modelo de crescimento exponencial, equação (1), para os conjuntos do híbrido OC 705 e CD 5501 nas duas épocas (Figura 2), para avaliação de cada híbrido.

Para testar a igualdade de equações, fixando-se os híbridos, ajustou-se a função do modelo de crescimento exponencial, equação (1), para os conjuntos de dados gerais (OC 705 e CD 5501) (Tabela 3). Para os dois conjuntos de dados gerais ajustados (OC 705 e CD 5501), as medidas de curvatura intrínseca indicaram presença de linearidade (Bates \& Watts, 1988). Apenas as medidas de curvatura paramétrica foram significativas. $\mathrm{O}$ viés de Box indicou que apenas o parâmetro B é responsável pelo afastamento da não linearidade (Ratkowsky, 1983). O p-valor para o teste de normalidade de Shapiro-Wilk indicou a não violação para todas as equações $(p>0,05)$ e o teste de Bartlett's para as equações completas indicou a não violação da homocedasticidade ( $>>0,05)$ (Tabela 3$)$.

As estatísticas F e Qui-quadrado para verificar se existe igualdade das equações, fixando o híbrido, apresentaram-se significativas para os híbridos OC 705 e CD 5501 (Tabela 4). Assim, o percentual de

Tabela 1. Medidas referentes ao ajuste dos dados de percentuais germinativos de dois híbridos na época um (E1) e na época dois (E2), pelo modelo $\mathrm{Y}=\mathrm{A}-\mathrm{B} \exp (-\mathrm{Ct})$.

\begin{tabular}{|c|c|c|c|c|c|c|c|}
\hline \multirow[t]{2}{*}{ Estimativas } & & \multicolumn{3}{|c|}{ Época 1 (E1) } & \multicolumn{3}{|c|}{ Época 2 (E2) } \\
\hline & & OC 705 & CD 5501 & Geral & OC 705 & CD 5501 & Geral \\
\hline \multirow{3}{*}{$\begin{array}{l}\text { Estimativa dos } \\
\text { parâmetros }\end{array}$} & A & 0,9704 & 1,0000 & 0,9875 & 1,0000 & 1,0000 & 1,0000 \\
\hline & B & 11,2660 & 7,5668 & 9,0923 & 4,7158 & 4,3049 & 4,4751 \\
\hline & $\mathrm{C}$ & 0,1124 & 0,0929 & 0,1021 & 0,0808 & 0,0718 & 0,0760 \\
\hline \multirow{3}{*}{ Viés de Box } & $\mathrm{A}$ & 0,0011 & 0,0031 & 0,0009 & 0,0014 & 0,0022 & 0,0032 \\
\hline & B & 0,0320 & 0,0425 & 0,0190 & 0,0152 & 0,0143 & 0,0265 \\
\hline & $\mathrm{C}$ & 0,0023 & 0,0035 & 0,0014 & 0,0014 & 0,0014 & 0,0025 \\
\hline Curv. Paramétrica & & 3,3528 & 3,4889 & 2,4520 & 1,9684 & 1,7272 & 2,4464 \\
\hline Curv. Intrínseca & & 0,0935 & 0,1145 & 0,0744 & 0,0711 & 0,0705 & 0,0950 \\
\hline Normalidade $(\mathrm{p}>0,05)$ & & 0,5807 & 0,0533 & 0,3666 & 0,5525 & 0,0523 & 0,4315 \\
\hline SQE & & 0,00518 & 0,00945 & 0,0177 & 0,00257 & 0,00322 & 0,0252 \\
\hline
\end{tabular}

Valor limite para o viés de Box $=0,01$.

Valor limite para a curvatura $=0,28$.

Tabela 2. Resultados do teste F e Qui-quadrado para a igualdade de equações de dois híbridos, nas duas épocas de semeadura.

\begin{tabular}{cccccc}
\hline Híbridos & Equações (geral) & $\chi^{2}$ & P valor & F & P valor \\
\hline Época 1 & $\hat{\mathrm{Y}}=0,9875-9,0923 \cdot \exp (-0,1021 . \mathrm{t})$ & 3,81 & 0,28 & 0,43 & 0,98 \\
\hline Época 2 & $\hat{\mathrm{Y}}=1-4,4751 . \exp (-0,076 . \mathrm{t})$ & 29,4 & 0,00 & 15,6 & 0,00 \\
\hline
\end{tabular}



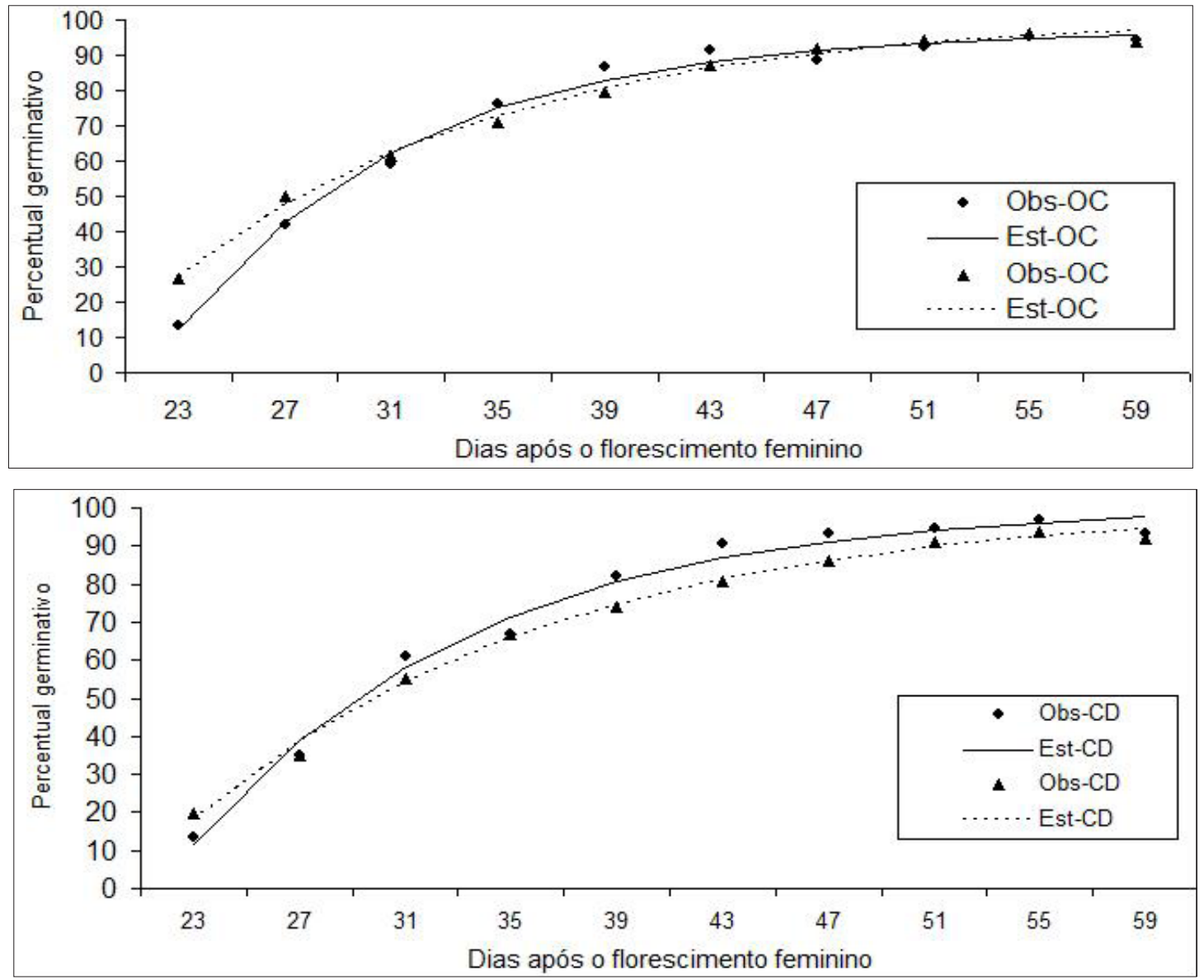

Figura 2. Porcentagem de plântulas normais obtidas no teste de germinação das sementes do híbrido OC 705 (A) e CD 5501 (B), em duas épocas de semeadura, em função da época de amostragem.

germinação das sementes do híbrido OC 705, semeado nas épocas 1 e 2 (E1 e E2), não pode ser representado nas duas épocas por uma única equação, devendo-se trabalhar com uma equação para cada época. $\mathrm{O}$ mesmo ocorreu para o híbrido CD 5501.

Outro ponto importante para recomendação à empresa de sementes é determinar o tempo (dias) de estabilização do percentual máximo de germinação para realização da colheita. A Tabela 5 indica, para cada híbrido e época, o tempo mais adequado de realização da colheita, ou seja, o tempo de estabilização do percentual de germinação das sementes de milho. Constata-se que, na primeira época de semeadura (E1), onde se obteve uma única equação para representar os dois híbridos, a equação geral forneceu o tempo de 57 dias para atingir o percentual máximo de $96,1 \%$ de germinação das sementes para os dois híbridos. Para a segunda época (E2) o híbrido OC 705 tem como tempo ideal de colheita 56 dias após o florescimento feminino, com percentual máximo de germinação das sementes de $95,3 \%$, e para o híbrido CD 5501 tem-se o tempo de 58 dias com percentual máximo de germinação das sementes de 93,3\%.

Isso é muito importante, pois as sementes devem ser colhidas o mais breve possível, a partir do momen- 
Tabela 3. Medidas referentes ao ajuste dos dados de percentuais germinativos de cada híbrido nas duas épocas de semeadura, pelo modelo $\mathrm{Y}=\mathrm{A}-\mathrm{B} \exp (-\mathrm{Ct})$.

\begin{tabular}{lccc}
\hline Estimativas & & Geral (OC 705) & Geral (CD 5501) \\
\hline \multirow{2}{*}{ Parâmetros estimados } & $\mathrm{A}$ & 0,9833 & 1,0000 \\
& $\mathrm{~B}$ & 7,4504 & 5,7593 \\
& $\mathrm{C}$ & 0,0978 & 0,0830 \\
\hline \multirow{2}{*}{ Viés de Box } & $\mathrm{A}$ & 0,0015 & 0,0023 \\
& $\mathrm{~B}$ & 0,0245 & 0,0230 \\
& $\mathrm{C}$ & 0,0021 & 0,0021 \\
\hline Curv. Paramétrica & & 2,8264 & 2,4084 \\
\hline Curv. Intrínseca & & 0,0890 & 0,0868 \\
\hline Normalidade $(\mathrm{p}>0,05)$ & & 0,7273 & 0,0501 \\
\hline SQE & & 0,0211 & 0,0249 \\
\hline
\end{tabular}

Valor limite para o viés de Box $=0,01$.

Valor limite para a curvatura $=0,28$.

Tabela 4. Resultados do teste F e Qui-quadrado para a igualdade de equações de dois híbridos, nas duas épocas de semeadura.

\begin{tabular}{cccccc}
\hline Híbridos & Equações (geral) & $\chi^{2}$ & $\begin{array}{c}\mathbf{P} \\
\text { valor }\end{array}$ & $\mathbf{F}$ & $\begin{array}{c}\mathbf{P} \\
\text { valor }\end{array}$ \\
\hline OC 705 & $\hat{Y}=0,9833-7,4504 \cdot \exp (-0,0978 . \mathrm{t})$ & 20,4 & 0,00 & 8,3 & 0,00 \\
\hline CD 5501 & $\hat{\mathrm{Y}}=1-5,7593 \cdot \exp (-0,083 . \mathrm{t})$ & 13,5 & 0,00 & 4,5 & 0,04 \\
\hline
\end{tabular}

Tabela 5. Tempo (dias) de ocorrência do percentual máximo de estabilização dos híbridos, nas duas épocas de semeadura.

\begin{tabular}{lccc}
\hline \multicolumn{1}{c}{ Híbridos } & Época & Tempo (dias) & \% germinação \\
\hline OC 705 & E1 & 56 & 95,0 \\
CD 5501 & E1 & 57 & 96,2 \\
Geral & & 57 & 96,1 \\
\hline OC 705 & E2 & 56 & 95,3 \\
CD 5501 & E2 & 58 & 93,3 \\
\hline
\end{tabular}

to em que atingem o percentual máximo de germinação, que é indicativo da máxima qualidade fisiológica, procurando evitar a permanência desnecessária das sementes no campo, o que frequentemente compromete seu desempenho, ou seja, acelera o processo de deterioração. Assim, essa metodologia estatística vem ao encontro do que a indústria sementeira necessita.
O Ministério da Agricultura, Pecuária e Abastecimento, por meio da Instrução Normativa $n^{\circ} 45$, de 17 de setembro de 2013, estabelece normas específicas e os padrões de identidade e qualidade para produção e comercialização de sementes, conforme divulga a Associação Brasileira de Sementes e Mudas, em que ficou estipulado para o milho o percentual 
mínimo de germinação das sementes igual a $85 \%$. As equações ajustadas forneceram os tempos necessários para se ter o percentual mínimo de germinação das sementes (Tabela 6) e indicaram que os híbridos OC 705 e CD 5501, na primeira época de semeadura (E1), representados por uma única equação, atingem esse percentual em menor tempo, ou seja, 41 dias após a antese.

Tabela 6. Tempo (dias) de ocorrência do percentual mínimo de $85 \%$ de germinação das sementes dos híbridos de milho, nas duas épocas de semeadura.

\begin{tabular}{lcc}
\hline \multirow{2}{*}{ Híbridos } & \multicolumn{2}{c}{ Tempo (dias) } \\
\cline { 2 - 3 } & E1 & E2 \\
\hline OC 705 & 40 & 43 \\
CD 5501 & 43 & 47 \\
Geral & 41 & \\
\hline
\end{tabular}

Em face das análises realizadas, é possível identificar os híbridos OC 705 e CD 5501 na primeira época como a combinação mais conveniente de híbrido e época de semeadura. Esse resultado indica que a época de semeadura tem mais influência que o tipo de híbrido no percentual de germinação das sementes.

\section{Conclusões}

A primeira época foi a mais adequada para a semeadura dos dois híbridos, devendo a colheita ser realizada 57 dias após o florescimento feminino, para se obter o percentual máximo de $96,1 \%$ de germinação.

Verificou-se que, fixando a primeira época de semeadura, os dois híbridos (OC 705 e CD 5501) podem ter o percentual de germinação descrito por uma única equação, indicando a semelhança deles quando cultivados na primeira época. Porém, um mesmo híbrido não pode ser representado por uma única equação para duas épocas diferentes de semeadura, indicando que o percentual germinativo de um mesmo híbrido não é semelhante em diferentes épocas de semeadura.

\section{Referências}

BATES, D. M.; WATTS, D. G. Nonlinear regression analysis and its applications. New York: John Wiley \& Sons, $1988.365 \mathrm{p}$.

BRASIL. Ministério da Agricultura, Pecuária e Abastecimento. Secretaria de Defesa Agropecuária. Regras para ánalise de sementes. Brasília, DF, 2009. $395 \mathrm{p}$.

CARVALHO, S. J. P.; CHRISTOFFOLETI, P. J. Influência da luz e da temperatura na germinação de cinco espécies de plantas daninhas do gênero Amaranthus. Bragantia, Campinas, v. 66, n. 4, p. 527-533, 2007.

DOI: $10.1590 /$ S0006-87052007000400001.

CARDOSO, V. J. M.; PEREIRA, F. J. M. Germinação de sementes de Drymaria cordata (L.) Willd. ex Roem \& Schult.: efeito do potencial hídrico. Revista Brasileira de Botânica, São Paulo, v. 31, n. 2, p. 253-261, 2008. DOI:10.1590/S0100-84042008000200008.

GAZOLA, S.; SCAPIM, C. A.; ARAÚJO, A. M. M.; ROSSI, R. M.; AMARAL JÚNIOR, A. T.; VIVAS, M. Nonlinear models to describe the maize seed quality during the maturation stage: a Bayesian approach. Australian Journal of Crop Science, v. 10, n. 5, p. 598-603, 2016. DOI: 10.21475/ajcs.2016.10.05.p6361.

GAZOLA, S.; SCAPIM, C. A.; BRACCINI, A. L.; ARAÚJO, A. M. M.; AMARAL JÚNIOR, A. T.; VIVAS, M. Probit regression to estimate the physiological potential of hybrid maize seed. Journal of Seed Science, Londrina, v. 37, n. 1, p. 33-39, 2015.

DOI: $10.1590 / 2317-1545 v 37 \mathrm{n} 1140984$.

GUIMARÃES, V. P.; RODRIGUES, M. T.; SARMENTO, J. L. R.; ROCHA, D. T. Utilização de funções matemáticas 
no estudo da curva de lactação em caprinos. Revista

Brasileira de Zootecnia, Viçosa, MG, v. 35, n. 2, p. 535543, 2006. DOI: 10.1590/S1516-35982006000200028.

NOGUEIRA, G. S.; LEITE, H. G.; REIS, G. G.; MOREIRA, A. M. Influência do espaçamento inicial sobre a forma do fuste de árvores de Pinus taeda L. Revista Árvore, Viçosa, MG, v. 32, n. 5, p. 855-860, 2008.

DOI: $10.1590 / \mathrm{S} 0100-67622008000500010$.

RATKOWSKY, D. A. Nonlinear regression modeling. New York: Marcel Dekker, 1983. 276 p.

REGAZZI, A. J. Teste para verificar a igualdade de parâmetros e a identidade de modelos de regressão nãolinear. Revista Ceres, Viçosa, MG, v. 50, n. 287, p. 9-26, 2003.

REGAZZI. A. J.; SILVA, C. H. O. Teste para verificar a igualdade de parâmetros e a identidade de modelos de regressão não-linear. I. dados no delineamento inteiramente casualizado. Revista de Matemática e Estatística, Jaboticabal, v. 22, n. 3, p. 33-45, 2004.
RIZZARDI, M. A.; LUIZ, A. R.; ROMAN, E. S.; VARGAS, L. S. Temperatura cardeal e potencial hídrico na germinação de sementes de corda-de-viola (Ipomoea triloba). Planta Daninha, Rio de Janeiro, v. 27, n. 1, p. 13-21, 2009. DOI: 10.1590/S0100-83582009000100003.

SÁNCHEZ-CORONADO, M. E.; COATES, R.; CASTROCOLINA, L.; BUEN, A. G.; PAEZ-VALENCIA, J.; BARRADAS, V. L.; HUANTE, P.; OROZCO-SEGOVIA, A. Improving seed germination and seedling growth of Omphalea oleifera (Euphorbiaceae) for restoration projects in tropical rain forests. Forest Ecology and Management, Wageningen, v. 243, n. 1, p. 144-155, 2007. DOI: $10.1016 /$ j.foreco.2007.03.001.

SIQUEIRA, E. M. D. L.; FONSECA, F.; PETERNELLI, S. L. A.; SALOMÃO, L. C. C. Método de comparação de modelos de regressão não-lineares em bananeiras. Ciência Rural, Santa Maria, v. 39, n. 5, p. 1380-1386, 2009. DOI: $10.1590 / \mathrm{S} 0103-84782009000500012$.

SOUZA, G. S. Introdução aos modelos de regressão linear e não-linear. Brasília, DF: Embrapa-SPI, 1998. 505 p. 